\title{
Research on trust mechanism of cooperation innovation with big data processing based on blockchain
}

\author{
Qi Liu ${ }^{1,2^{*}}$ and Xiao Zou²
}

\begin{abstract}
In recent years, the status of cooperation and innovation has become increasingly important. Research on the behavior of production, education, and research organizations has attracted widespread attention. Blockchain technology is considered as another subversive technology following cloud computing, wireless sensor networks, and big data. It is highly concerned by governments, financial institutions, and technology companies. Blockchain technology is essentially a technical solution that collectively maintains a reliable database through a decentralized, high-trust way. Blockchain WEB technology has not only used in financial and other fields, but also has a great potential for application in the fields of production, education, and research. It has expected to play an important role in the establishment of a trust mechanism in the "Internet + " production, research, and innovation and promote education. Drawing on the experience in blockchain applications in the financial sector, the blockchain in the education sector mainly embodies six application modes: building individual science credit data, creating an intelligent education Taobao platform, developing a degree certificate system, and building a new ecology of open educational resources. Of course, due to the uniqueness and complexity of the trust field in the cooperation innovation of industry, universities, and research institutes, the application of blockchain technology is also faced with difficulties in the application, promotion, and operation of production and research, fuzzy ownership of educational big data, limited data storage space, and potential safety problems of blockchain technology. There are many challenges such as the privacy protection risks of teachers and students.
\end{abstract}

Keywords: Cooperation innovation, Internet +, Blockchain WEB, Big data, Sensor network

\section{Introduction}

Various types of innovation have brought about tremendous economic development in various countries and have contributed to the progress of human society. Looking at the world's leading companies, it is because of their active promotion of innovation that they will never fail and even come to the forefront of the world's enterprises. It can be said that technological innovation is the source of human wealth and the fundamental driving force for economic growth. Technological innovation can increase the benefit of products and reduce the cost of resources, which is the core strength of improving the competitiveness of enterprises [1]. Lassar believes that technology can achieve the effect of multiplication of

\footnotetext{
* Correspondence: liuqi197711@163.com

'Business School, Central South University, Changsha 410079, China

${ }^{2}$ Business School, Hunan University of Technology, Zhuzhou 412000, China
}

value through sharing, and cooperation innovation of enterprise technology is one of the most effective ways to achieve technology sharing and is a reliable guarantee for enterprises to establish a sustainable competitive advantage. It will become a kind of technology innovation of many enterprises [2]. Collaborative innovation has usually based on the common interests of partners, and it has based on the premise of sharing resources or complementing each other. It has clear objectives for cooperation, cooperation deadlines, and cooperation rules. All parties involved in joint ventures invest in the whole process or some aspects of technological innovation: participate in, share results, and share risks. Collaborative innovation is generally concentrated in emerging technologies and high-tech industries, and $R \& D$ is the main form of cooperation [3]. Currently facing the challenges of knowledge economy and technological progress, many countries 
are committed to the construction of a "national innovation system." British scholar Freeman first proposed the concept of a "national innovation system," which consists of companies, universities, research institutes, and intermediaries. In addition, enterprises are the main body of innovation. Universities and scientific research institutions are the source of innovation. At the same time, they provide and train innovative talents. Intermediary agencies are an important way to communicate and promote knowledge flow and technology diffusion. Governments create policies and laws that encourage and guide innovation [4]. Cooperation innovation between industry, universities, and research institutes has considered as an important means to promote science and technology, education, and economic progress. At present, with the integration of science, technology, and economy, the cooperation between industry, universities, and research institutes around the world is showing a trend of continuous innovation, scale expansion, continuous improvement of the level, more flexible mechanisms, and more dynamic environment [5]. Under the new and complicated market environment, the cooperation between production, education, and research is inefficient or even unsuccessful. It is the result of the combined effects of multiple factors, but the most fundamental reason lies in the mistakes made by the parties in the selection of models.

Blockchain technology is regarded as another subversive technology following cloud computing, Internet of things, and big data. It is highly concerned by governments, financial institutions, and technology companies. In 2016, developed countries such as the USA, the UK, and Japan have successively raised the blockchain technology to the national strategic level and established a blockchain development alliance. In the same year, the "Thirteenth Five-Year Plan for National Normalization" issued by the State Council for the first time included blockchains in Chinese national normalization plan and made it one of the strategic frontier technologies [6-8]. At present, all occupations have begun to pay attention to blockchain technology and actively explore the use of this technology to crack down on industry problems and promote innovation and development of the industry. In the financial sector, the global bank giants formed the R3 alliance, including more than 40 large international financial institutions such as HSBC, UBS, and the US Banking to develop blockchain technology. The US Nasdaq took the lead in introducing Linq, a security trading platform based on blockchain technology, which became an important milestone in the decentralization trend of financial and security markets. Zhou Xiaochuan, the governor of the People's Bank of China, pointed out at the digital currency seminar held in Beijing that it is necessary to pay close attention to the impact of blockchains and other technologies on the financial sector and demand the exploration of the issuance of digital currencies.

With the gradual improvement of blockchain technology and the continuous expansion of application fields, some international educational institutions and scholars have begun to pay attention to and explore the application of blockchain technology in the field of education. Prof. Qing Li from Beijing University of Posts and Telecommunications pointed out that blockchain technology has great application potential in the field of education, which helps to create a more open and more credible education system. As a whole, the current application of blockchain technology in the field of education has just begun. It lacks mature application cases and clear application ideas. It urgently needs to strengthen the collaborative research and practice of production, learning, and research. Based on this, this article will introduce the concept and core technology of blockchain technology, analyze the experience and enlightenment from blockchain application in the financial field, and discuss the educational application ideas and values of blockchain technology in combination with the existing typical education cases $[9,10]$. We analyze possible challenges to promote the application of blockchain technology in education.

\section{Methods}

\subsection{Blockchain WEB of big data}

Blockchain is a shared ledger technology, and any participant in a commercial network can view transaction system records (accounts). Blockchain is a distributed multi-node "consensus" implementation technology. Through the blockchain, the entire process of value transfer (transaction) can be recorded completely. The formation of blockchain has connected in chronological order. Each node participating in consensus formation has a complete copy of the blockchain information. In the blockchain trust mechanism, all members participate in the supervision, control, and audit of the trust value and perform password protection for the whole lifecycle for almost all the elements of data records such as addresses, chains, public keys, private keys, and abstracts. In addition, it is global and random. An alliance chain, which is equivalent to the sum of a global super password, switch, key signature server, and password book, is a continuous credit. Second, never take the opportunity. Because of the member's dynamic computing mechanism, all member nodes have synchronized in real time, and local control cannot be achieved. The global nodes are randomly distributed and correspondingly controlled under the control of the operation clock and dynamic time stamp and never shutdown, and the credit is continuous [11]. The technical characteristics of strong blockchain trust have derived from the lack of 
central security responsibilities of its node members. The local collaboration of members achieves the overall supervision of system security, integrates supervision and processes, and realizes a positive feedback mechanism for security in systems science. Under the current calculation conditions, it is not possible to pass the reliable overstepping of dynamic time stamps, and the credits are continuous. Figure 1 shows the blockchain WEB of big data.

\subsection{Trust mechanism of WEB}

In social activities, people usually evaluate the reliability of trading activities of WEB based on the historical records of their direct transactions or friends' recommendation information and decide whether to conduct transactions based on the evaluation results. In a peer-to-peer network environment, trust and the credibility problem to solve is similar to the reliability evaluation in social activities. Before the transaction, with the help of the trust mechanism, both parties can understand each other's credibility, thus increasing the safety factor of the transaction and avoiding potential safety hazards in the transaction process. The main contents of the trust and reputation mechanism include collecting historical transaction records between nodes in the system, calculating the credibility of each node based on the collected transaction records, and determining whether to conduct transactions based on the credibility of the nodes. The main points of the study are as follows: (1) The representation method of trust and reputation describe how to express the trust and reputation of the node in the system: It is the core component of the trust and reputation mechanism research; (2) The calculation method of trust and reputation: Transaction information assesses the degree of trust or reputation; and (3) The storage of trust and reputation values: How the calculated node credibility is stored in the system is related to how to obtain the trust and reputation value of the node. This paper will introduce the latest research results of the trust or reputation mechanism in the peer-to-peer network based on the research points of the trust and reputation mechanism.

At present, there is no unified definition of trust. This article [12] comprehensively describes the descriptive definition of trust and some properties of trust and lists some basic concepts related to trust.

Definition 1. Trust is a kind of subjective judgment based on existing knowledge. It is a measure of subject B's ability to provide specific services (or perform specific actions) according to the environment of subject $A$ according to the intention of subject $A$. Definition 2. Direct trust is the trust of subject $B$ derived from subject A's direct transaction history with subject B.

Definition 3. Recommendation trust is the trust formed by the subjects based on the recommendation of a third party. It is also called indirect trust.

Definition 4. The trust degree is a quantitative representation of trust, also known as credibility.

Figure 2 describes the relationship between the above definitions. The direct trust degree of entity A for entity $\mathrm{B}$ is denoted as $T_{\mathrm{AB}}$, and the direct trust degree of entity $\mathrm{B}$ for entity $\mathrm{C}$ is denoted as $T_{\mathrm{BC}}$. Since there is no direct relationship between entity $\mathrm{A}$ and entity $\mathrm{C}$. In the historical record of the transaction, entity A needs to request assistance from entity B to obtain the credibility of entity C. According to the recommendation of entity B, entity A can obtain recommendation trust $T_{\mathrm{AC}}$ of entity $\mathrm{C}$.

Reputation is a concept closely related to trust, but reputation and trust are different. In order to illustrate

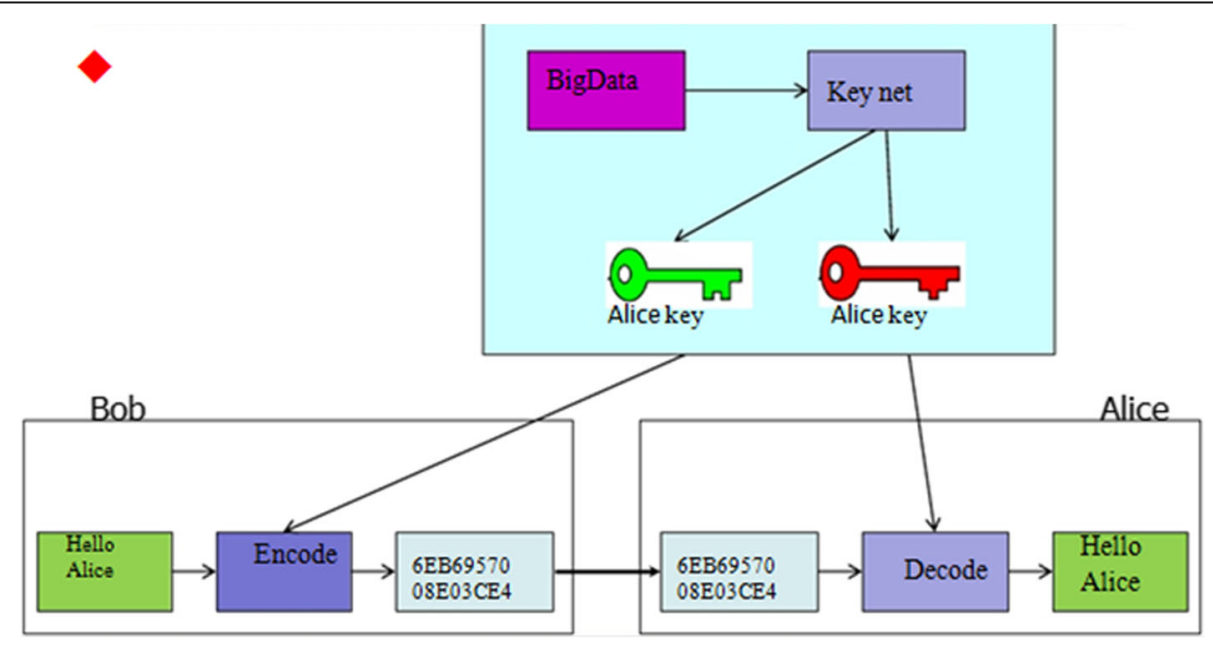

Fig. 1 The blockchain WEB of big data 


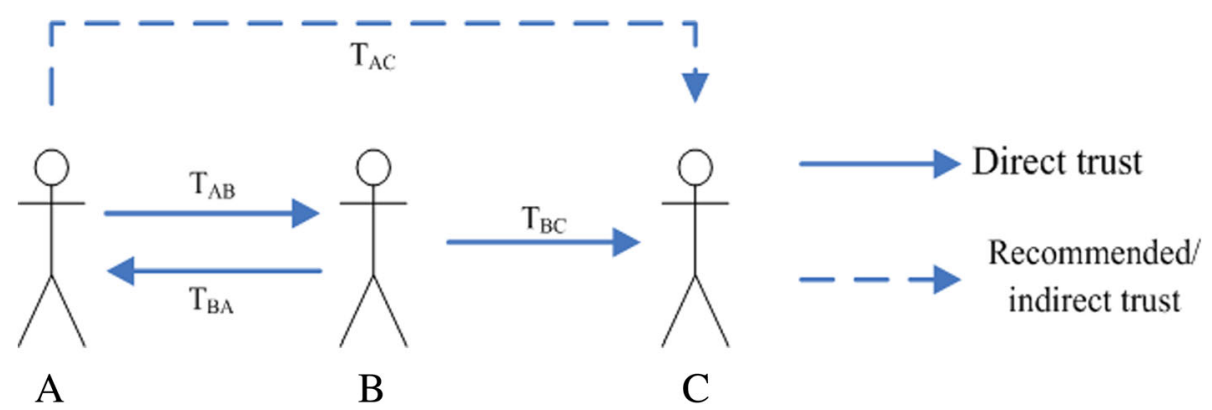

Fig. 2 Relationship between mutual information and information entropy in WEB

the connection and difference between the two, the following attempts to give a definition of reputation in peer-to-peer networks. Reputation is a comprehensive measure of the quality or characteristics of a node's existing services. It reflects the level of performance of a node's promised service and the trust of other nodes in the network. Figure 3 shows trust and reputation mechanism in WEB-P2P file sharing system.

From this, it can be seen that trust is active and it is an evaluation of a subject's ability to another subject, based on the evaluation of historical transactions; while reputation is passive, it is through the historical behavior of interaction or resource sharing to predict the user, whether the behavior is credible [13] and reputation is a collection of trusted information. Reputation is a global concept and has objectivity. It is the total of all subjects in the network to evaluate a subject. Trust is a local concept and has subjectivity. It only happens between two entities. Trust depends to a certain extent on reputation, but it has not determined by reputation. Figure 3 shows the relationship between trust and reputation mechanisms in the P2P file sharing system listed in [14]. In the figure, the FP (file provider) is a file provider. From the figure, you can see the implementation process of the trust and reputation mechanism and the relationship between them. A peer wants to choose a trusted FP if historically his experience with interacting with FP has found a FP with the highest level of trust in his trusted FP database to interact with; if he has not previously had experience with interacting with FP or has little knowledge of FP, he is from other peers. In the recommendation, we can calculate and select a FP with a high reputation value to interact. Through the satisfaction of this interaction, the FP has evaluated, and the credibility of the FP has updated. At the same time, the credibility of the peers providing the recommendation has updated. There is a premise that the credibility of FP with high reputation value is also high.

\section{Experimental}

3.1 Application mode of blockchain WEB technology From the perspective of the development of blockchain technology, finance is the first area of application and

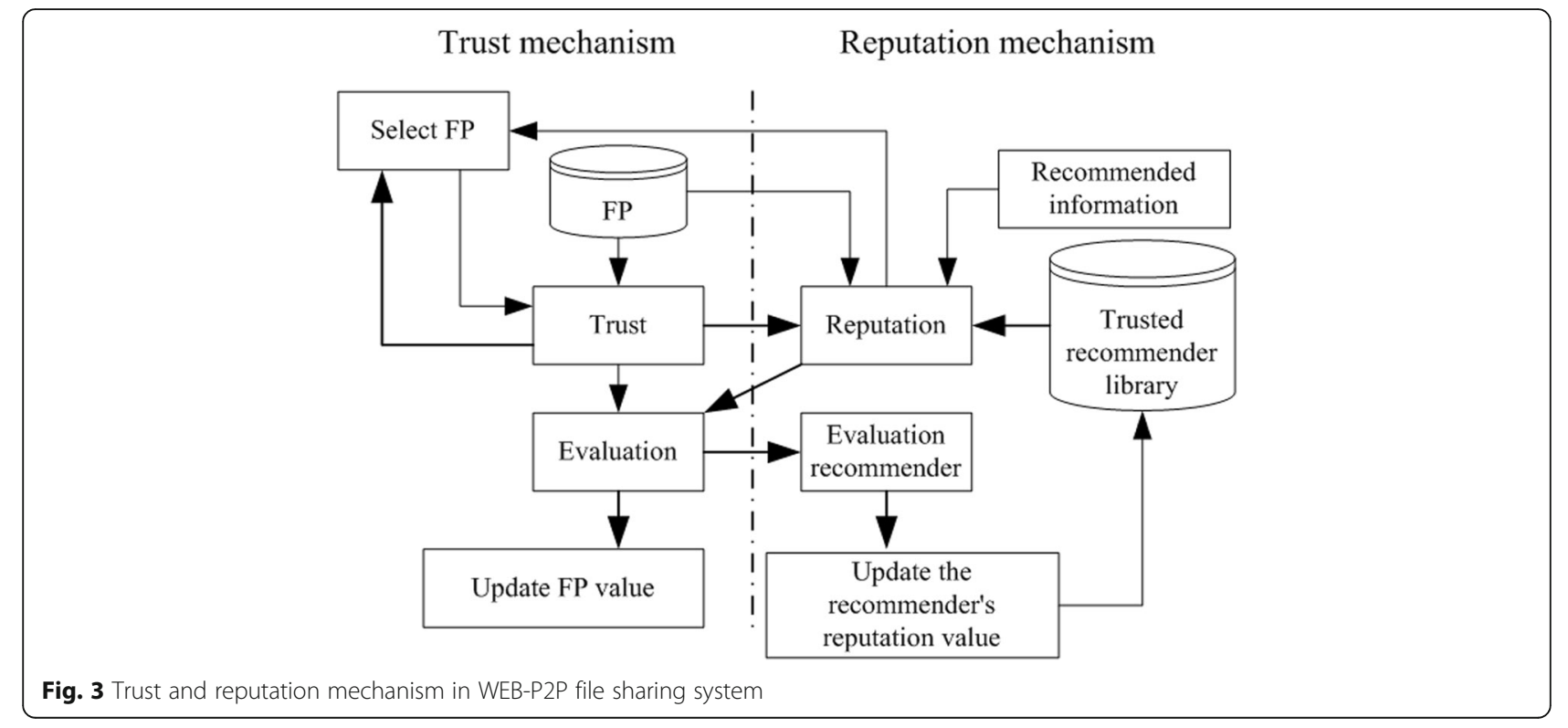


development. Currently, many international blockchain application cases also come from the financial field. Blockchain technology is highly concerned and enthusiastic by venture capitalists. It has gradually become a hot topic in the field of global innovation and is setting off a new round of capital war. A number of world-renowned financial institutions have jointly established blockchain technology application alliances such as R3 CEV and Hyperledger to cope with the technical standard competition and disruptive innovation wave in the financial sector. "The characteristics of transparency and data tamperability of blockchain systems are fully applicable to student credit management, career advancement, academics, proof of qualifications, industry-university cooperation, etc., and have important value for the healthy development of education and employment." The survey found that the current application of blockchain technology at home and abroad is in an embryonic state, and a few educational institutions have actively explored. Internet + education is the general trend of global education development and transformation. Its purpose is to use the Internet thinking, technology, and models to transform the traditional education ecosystem and achieve structural changes in the education system. Blockchain technology has expected to play an important role in the construction of Internet educational ecology. The value and ideas of its educational application have mainly reflected in six aspects: the establishment of an individual school letter data, the creation of an intelligent education Taobao platform, the development of a degree certificate system, and the construction of Open the new ecology of educational resources, realize the "self-organization" of online learning communities and develop decentralized education systems. Figure 4 shows a new Internet + education ecosystem based on blockchain technology. Blockchain technology helps promote the reform of the education system and accelerates the evolution of the education system.

There are two kinds of nodes in the entire OBC network, validating peer and non-validation peer. The former is a full-featured node and forms a fully connected topology. The latter is a proxy node and is attached to a neighboring authentication node. An application program can be connected to a verification node or a non-authentication node.

A key management nodule is required in the $\mathrm{OBC}$ network. Considering the $3 \mathrm{f}+1$ consensus mechanism of the PBFT algorithm, a typical fault tolerance (allowing one node failure) environment requires four validation peer nodes. If there is only one VP node in the OBC network (extreme simplification), the application can directly connect with this node and execute the Chaincode code on it. In this case, the VP node only needs accounting and no consensus is required. If there are multiple VP nodes in the OBC network, the application program can indirectly access the VP through the NVP node (the NVP and the application can be one-to-many). At this time, the VP node needs to verify the transaction and run. Figure 5 shows the single-node and multi-node networks of blockchain WEB.

\subsection{Trust mechanism of blockchain network}

The purpose of network security is to provide a protection mechanism to prevent the protected subject from being attacked and illegally accessed by a malicious subject. In the traditional network security mechanism, the protected subject refers to the service provider, and the malicious subject generally comes from the service requester. This security mechanism has called hard

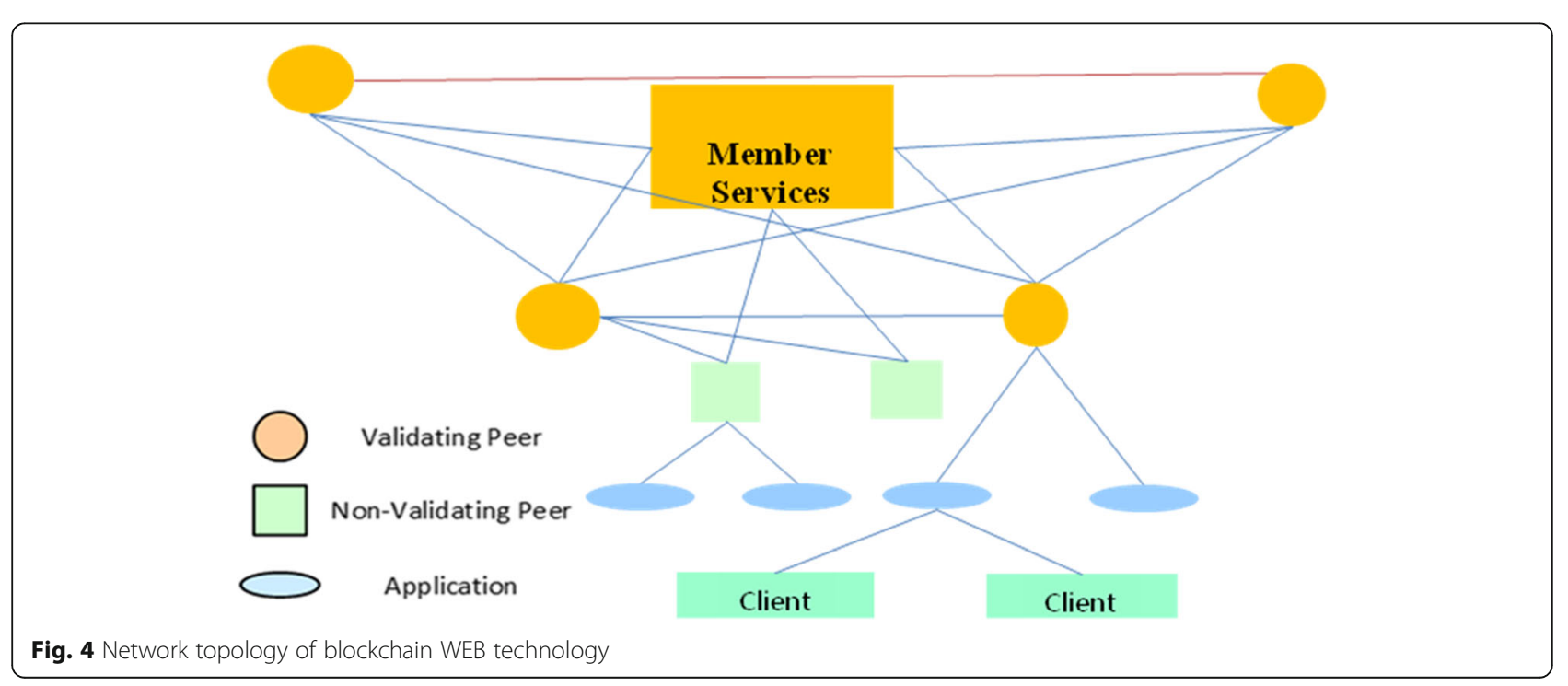




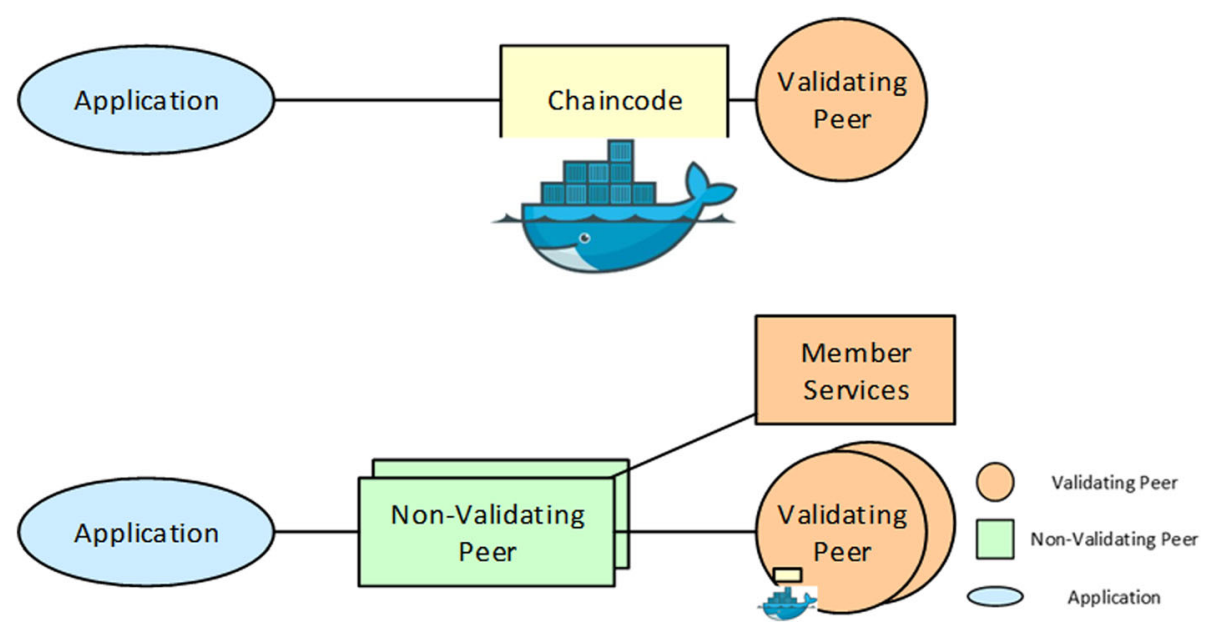

Fig. 5 Single-node and multi-node networks of blockchain WEB

security in the literature [15]. The trust mechanism provides the security protection measures which are obviously different from those provided by traditional security mechanisms. The file sharing system (FR) selects the most trusted one among multiple candidates FPs when downloading a file. FP is used as a download source. It can be seen that the trust mechanism is mainly to protect the security of FR, to avoid FR download to malicious files. The protected subject in the trust mechanism is the service requester; potential malicious users are the service providers. The security protection measures provided by the mechanism have referred to as soft security [16].

In the blockchain network, some trusted nodes are selected as the core node (Credit Node). Others are light nodes (terminal nodes TN Terminal Node). The CN node constructs an AS (Autonomous System). The CN core hotspot The synchronization protocol BSP (Block Synchronization Protocol) is used to build a core circle. The nodes in the core circle form a mesh network, synchronize information in real time, and jointly maintain a global blockchain. The core nodes determine the online through handshaking. $\mathrm{CN}$ core nodes can join or exit in real time. The newly added core node first establishes a neighbor relationship with other hotspots, synchronizes them, and obtains a global blockchain. After synchronization is completed, it can accept association requests of light nodes. The TN node obtains a list of core nodes through DNS. The algorithm first tries to communicate with several core nodes in real time through an algorithm. A list of hotspot nodes is established based on the communication delay, and a neighbor relationship with the first priority hotspot is established. If it is not successful, then analogously, if the contact with the $\mathrm{CN}$ hotspot is interrupted, the next $\mathrm{CN}$ hotspot is immediately looked for. The light node can change the access hotspot at any time and adhere to the principle of peer-to-peer peering. The upgrade of the hot-core node itself does not affect the light node and manages the entire blockchain system. Figure 6 shows the trust mechanism of blockchain network synchronization scheme.

There is also a link between the traditional security mechanism and the trust security mechanism. Computers or network systems protected by traditional security mechanisms are not vulnerable to attacks by malicious nodes, and the possibility of internal malicious programs (such as Trojan horses) has reduced. The credibility will be high; on the contrary, the credibility of those nodes that have not protected by the security mechanism will be low. In addition, there are trust mechanisms in the traditional security mechanisms, mainly relying on trusted third parties for identity authentication. The mechanism protects the security of the network system from different angles, and the two complement each other.

\subsection{Blockchain network for industry-university-research cooperation}

The demand for cooperation between production, education, and research is generated by the traction of market demand or the government's macro-guidance. Therefore, the cooperation model of industry, university, and research can have divided into two types as "demand for market demand" and "government macro-guidance" [17]. (1) The market demand traction cooperation model refers to the cooperation of industry, universities, and research institutes with the constraints of their own resources and market demands and other internal factors, and the cooperation mode between industry, universities, and research institutes in order to meet the demand of combination of technology and economy. According to 


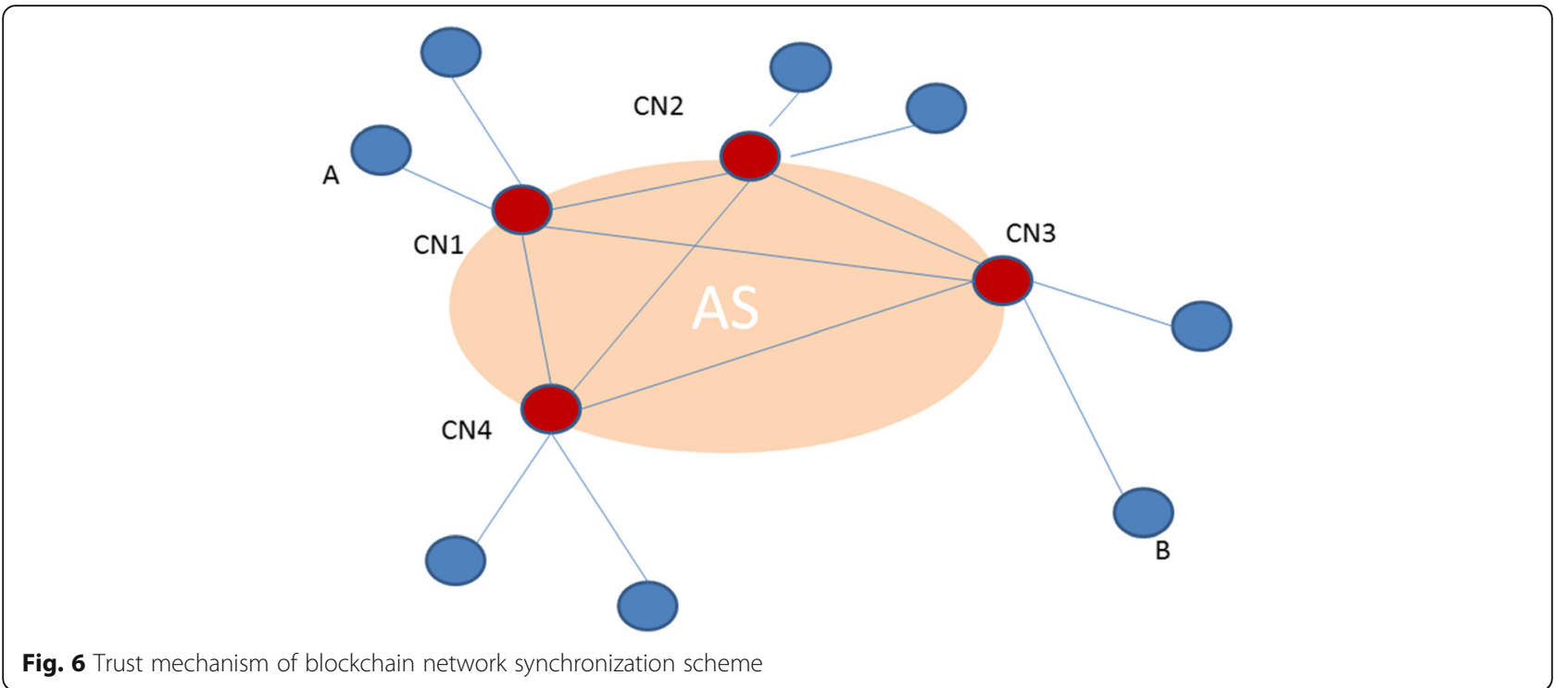

the classification of technology circulation and service, the main cooperation modes include technology transfer, technology development, technical consultation, and technical services. (2) The macro-guided cooperation model of the government and the macro-guided cooperation model of the government refer to the high-level cooperation between industry, universities, and research institutes at all levels in order to enhance national and local technological and economic strength and accelerate the development of high-tech fields. According to the form of the combination of technology and economy, the common modes of cooperation mainly include joint scientific and technological research, co-construction engineering technology center, and co-construction university science park and high-tech industrial development zone. Any cooperation model has guided by the policies of the government's competent authorities, creating a locally optimized internal and external environment for high-tech integration bases, and actively attracting industry-university-research cooperation partners to bring projects to the park for entrepreneurial development to facilitate the acceleration of high-tech commercialization. The process of industrialization and internationalization has strengthened the strength of the local economy.

\subsubsection{Trust calculation (inference) method of blockchain WEB}

Trust calculation (inference) method refers to the method of calculating (speculating) the trust degree of the subject based on the collected evaluation data. The calculation (speculation) method does not depend on the trust value. The common calculation methods in the literature are the weighted average method, Bayesian method, fuzzy reasoning method, and gray reasoning method.

\subsubsection{Weighted average method of blockchain WEB}

At present, most trust mechanisms adopt this method, including some classic trust systems such as eBay, Credence, and Eigentrust. This method draws on the trust evaluation method among people in social networks, and its calculation method is as shown in formula (1) as follows:

$$
T_{i, j}=\alpha \cdot\left(\beta \cdot R_{d}+(1-\beta) R_{r}\right)-\gamma R_{i}
$$

where $T_{i, j}$ denotes the trust value of subject $i$ for subject $j, R_{d}$ is the direct trust value calculated based on the direct transaction record between subject $i$ and subject $j$, and $R_{r}$ is the indirect trust calculated by subject $i$ based on recommendation information of other subjects. For the value, $R_{i}$ is the risk value brought by the transaction, and $\alpha, \beta, \gamma$ represent different coefficients.

\subsubsection{Maximum likelihood estimation of blockchain WEB}

Maximum likelihood estimation (MLE) is a probability-based trust inference method, which is mainly applied to probability models and belief models. When the probability distribution of trust is known and the parameters of the probability distribution are unknown, MLE speculates on these unknown parameters based on the transaction results obtained. The inferred parameters make it most likely that these results will appear. If the probability distribution of trust is $p(x)$, the credibility of subject $i$ is $t_{i}$, and the probability of honest recommendation of subject $i$ is equal to its credibility, 
the transaction result with subject $j$ is $x_{i}, j$, and the neighbor node of subject $i$ is recorded as $n(i)$, then the MLE speculation method can be reduced to solving the maximum value of expression (2).

$$
\max _{t_{i}} \arg \log \prod_{k \in n(i)} p\left(x_{i, k}, t_{i}, l_{k}\right)
$$

\subsubsection{Bayesian method of blockchain WEB}

The Bayesian method is a result-based posterior probability estimate that is applicable to probabilistic models and belief models. Unlike MLE, it first assigns a priori probability distribution to the parameters to be inferred (prior probability) and then use the Bayes' rule to estimate the posterior probability of the parameter based on the outcome of the transaction. Based on the number of possible outcomes for the transaction evaluation, the prior probability distribution for the parameter to be speculated is divided into two. Species: Beta distribution and Dirichlet distribution, where beta distribution is only suitable for the case of binary evaluation results and is a special form of Dirichlet distribution. Due to space limitations, only the reasoning methods based on Dirichlet distribution are introduced here.

The hypothesis is that the evaluation has $k$ results. The prior probability distribution for each result is a uniform distribution, i.e., the probability of each occurrence. For $1 / k$, there are $n$ transactions in total, and each transaction gives an evaluation, where $i(i=1,2, \ldots, K)$ evaluations occur times $m_{i}\left(\sum m_{i}=n\right)$. The posterior probability distribution of parameter $p$ is as follows.

$$
f(p, m, k)=\frac{1}{\int_{0}^{1} \prod_{i=1}^{k} x^{\left(m_{i}+C / k-1\right)} d x} \prod_{i=1}^{k} p_{i}^{\left(m_{i}+C / k-1\right)}
$$

Among them, $C$ is a preset constant, and the larger $C$ is, the smaller the evaluation result is to the expected value of the parameter $p$, and $C$ is generally selected as $k$. The Bayesian estimation expectation value of the evaluation probability of the $i$ th evaluation result is as follows.

$$
E\left(p_{i}\right)=\frac{m_{i}+C / k}{C+\sum_{i=1}^{k} m_{i}}
$$

\subsubsection{Fuzzy reasoning method for blockchain WEB}

The fuzzy reasoning method is mainly applicable to the fuzzy trust model. Figure 7 is the general framework of fuzzy reasoning described in the literature and divided into three processes: fuzzification, fuzzy reasoning, and anti-fuzzification. The fuzzification process uses the membership function to synthesize evaluation data. Judgment is classified into fuzzy sets. Fuzzy reasoning infers trust relationships between subjects based on fuzzy rules or the fuzzy sets to which the subject's credibility belongs. Examples of inference rules are as follows.

IF the weighted trustworthiness value is high

AND the opinion weight is high

AND the opinion credibility is high

THEN trustworthiness level is high

Formal inference rules can be found. The anti-fuzzification reasoning results can be used to obtain the credibility of the subject.

\subsubsection{Gray system method for blockchain WEB}

The gray system theory is the theory proposed by scholars of China to study the control and decision problems of incomplete parameters systems, and it has been widely used in many industries. The literature proposed a credit report based on the gray system theory. Mechanism, but the current research on the trust mechanism, based on the gray system theory is not much. The reasoning process based on the gray system theory is shown in Fig. 8.

In the gray inference process, firstly, the gray relational analysis (gray relational analysis) is used to analyze the

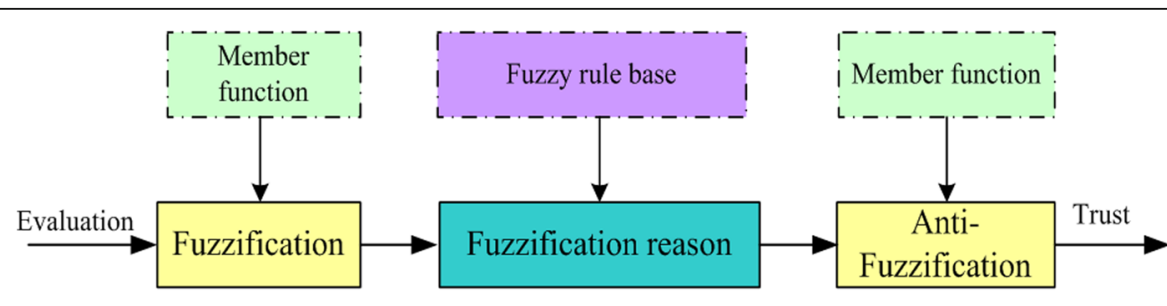

\begin{tabular}{|llll}
\hline Accurate input & Fuzzy input & Fuzzy output & Accurate output
\end{tabular}

Fig. 7 The basic framework of fuzzy reasoning 
evaluation result, and the gray relational degree (i.e., the evaluation vector) is obtained; if the evaluation involves multiple key attributes (such as in a file sharing system, the evaluation of the subject may involve the quality of downloading files, attributes of download speed, etc.), determining the weight relationship between attributes; calculating the whitening matrix using the whitening function and the evaluation vector; and calculating the clustering vector from the whitening matrix and the weight matrix, and clustering the vector response, the relationship between the subject and the gray level in the gray level set, and the clustering analysis of the clustering vector can obtain the gray class to which the subject belongs.

\subsection{Blockchain WEB results and discussion}

Chinese universities and affiliated scientific research institutions have $70 \%$ of high-tech achievements in China and are an important support for technological innovation. However, the long-term separation of enterprises and universities has resulted in a large number of scientific and technological achievements that have not translated into actual productivity. With the establishment and improvement of the market economy system, the national science and technology system reform requires that scientific research institutes and colleges, and universities face economic construction, accelerate the transformation and promotion of results, and make cooperation between industry, universities, and research institutes innovative. To solve the combination of science and technology and economy, science and technology provide services for economic construction, and science and technology have found an effective way to guide economic development. Figure 9 shows the workflow of R\&D blockchain WEB.

Taking Jiangsu Province's innovation in cooperation with industry, universities, and research institutes as an example, Jiangsu Province has 115 ordinary higher education institutions, 403 research institutes in universities and colleges, and 299 independent research and development institutions in government departments. These scientific research institutions are knowledge producers and talent cultivation bases for relevant applied research and important common technologies in Jiangsu Province and should play a leading role in Jiangsu's technological innovation activities. At present, Jiangsu Province is facing a new situation of upgrading industrial structure and improving independent innovation capabilities. Under the guidance and supervision of the provincial government, can universities, research institutes, and enterprises implement effective cooperation models and lead Jiangsu's cooperative innovation activities? Do you have the necessary conditions for innovation? How is its innovation performance? This will have practical significance for the construction of disciplines in Jiangsu Province.

From Fig. 10 below, it can be seen that from 2012 to 2015, the R\&D expenditures of large and medium-sized industrial enterprises in Jiangsu Province continued to increase, and the increase was large, exceeding $40 \%$ over the years, and the expenditure was much higher than that of research institutes and universities. The R\&D expenditure of scientific research institutes is lower than that of enterprises higher than institutions of higher learning. In 2013, the growth rate was $18 \%$ from the previous year. The growth rate in 2014 was modest, but it was followed by a downward trend. R\&D expenditures in colleges and universities were the lowest in terms of internal expenditures, and they also increased between 2012 and 2015, but the growth rate was far lower than that of enterprises. Except 2014, which was a 32\% increase from the previous year, the rest was below $15 \%$. According to the above analysis, from the perspective of the cooperative entity, enterprises in Jiangsu Province play a leading role in the process of cooperation between production, education, and research. This model is in

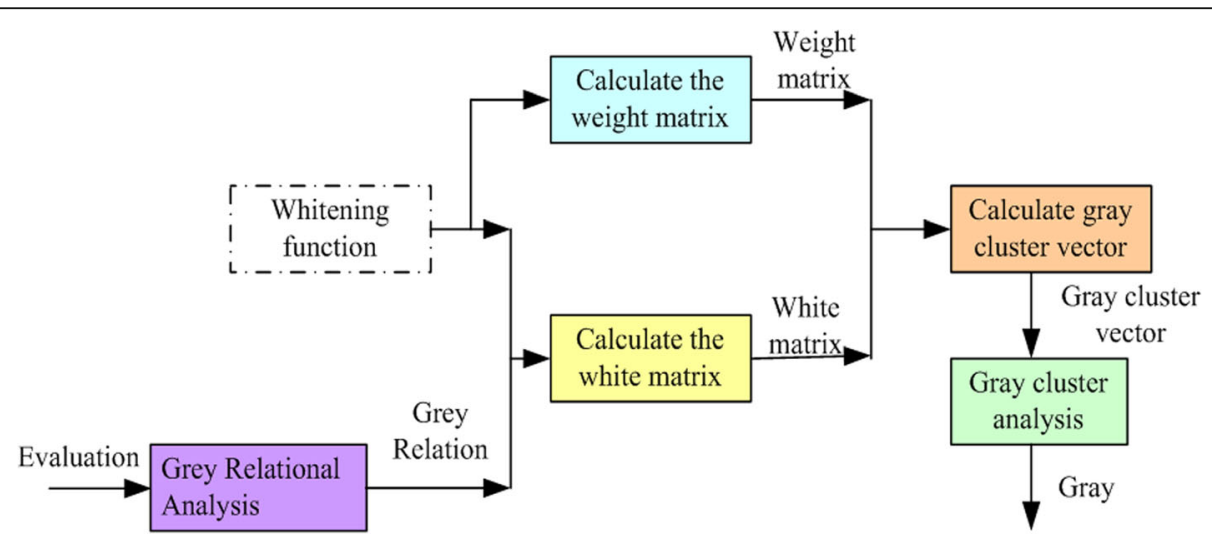

Fig. 8 The basic framework of gray reasoning 


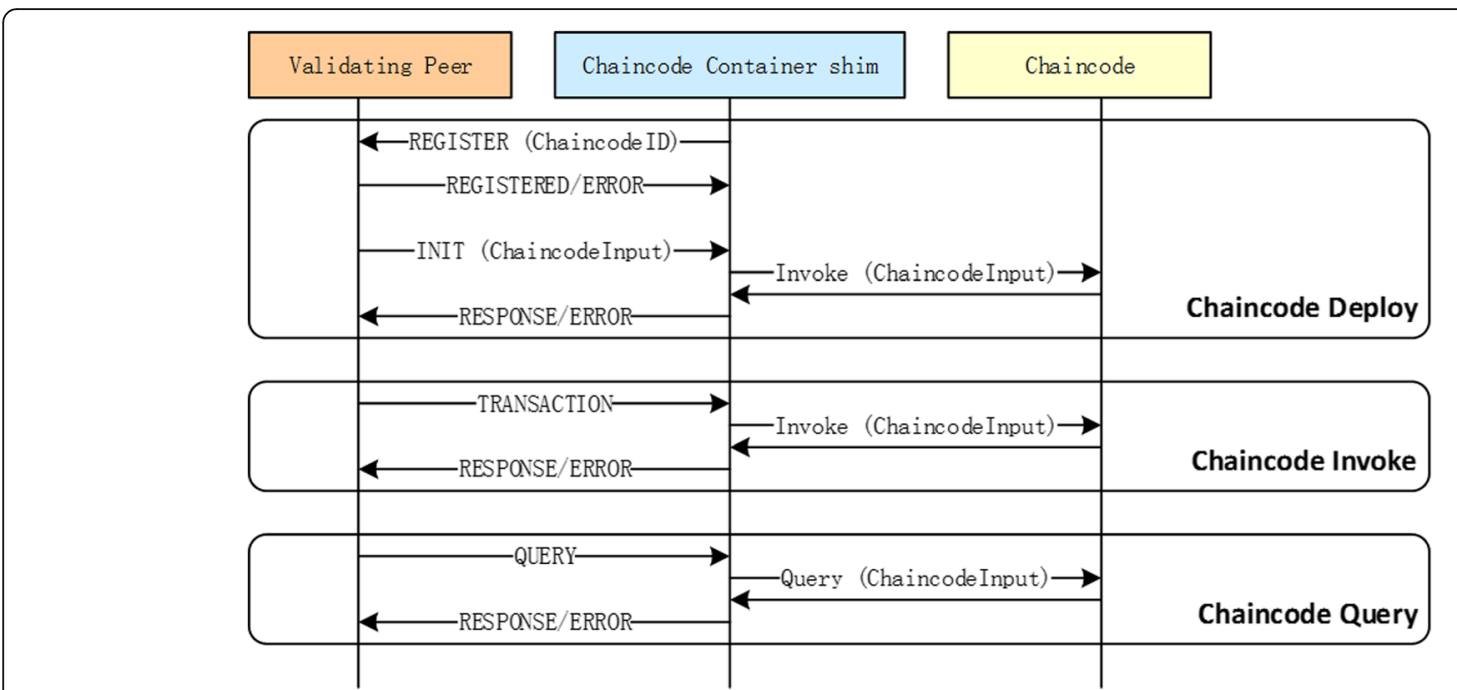

Fig. 9 Workflow of R\&D blockchain WEB

line with the needs of Chinese policies and economic development. Scientific research institutions, and colleges and universities, as the source of knowledge for technological innovation, the main undertaker of knowledge innovation, and the talent cultivation base, should increase the expenditure of internal funds and promote the development of science and technology to lead the economy.

\section{Conclusion}

Many experts and scholars have expressed their opinions on the selection of cooperative innovation models. Judging from the current research results, the cooperation and innovation of the industry-university-research cooperation innovation model and supervision have been a trend of cooperation and innovation in recent years. And it has promoted the intellectual resources of universities and scientific research institutes flow, or enterprises, enabling them to become the main body of technological innovation and to realize the sharing of risks, mutual benefit, mutual complementarily, common development, and optimization of social resources among all partners. The successful cooperation between industry, universities, and research institutes and the effective realization of the transformation of scientific and technological achievements are the key. Therefore, the cooperation relationship between the cooperation partners in the cooperation of industry, universities, and research institutes, the principal-agent relationship, and the government's supervision and other economic behavior choices should lead to more experts and scholars. In addition to use cases and empirical methods, we can also use more advanced game theory economic analysis methods, assuming parameters, modeling, and computer simulations, in order to depict real economic behavior selection patterns more vividly. Although we have achieved some achievements and experience in the joint work of Chinese domestic research and development, there are still many problems. At present, the research on the trust mechanism under P2P environment

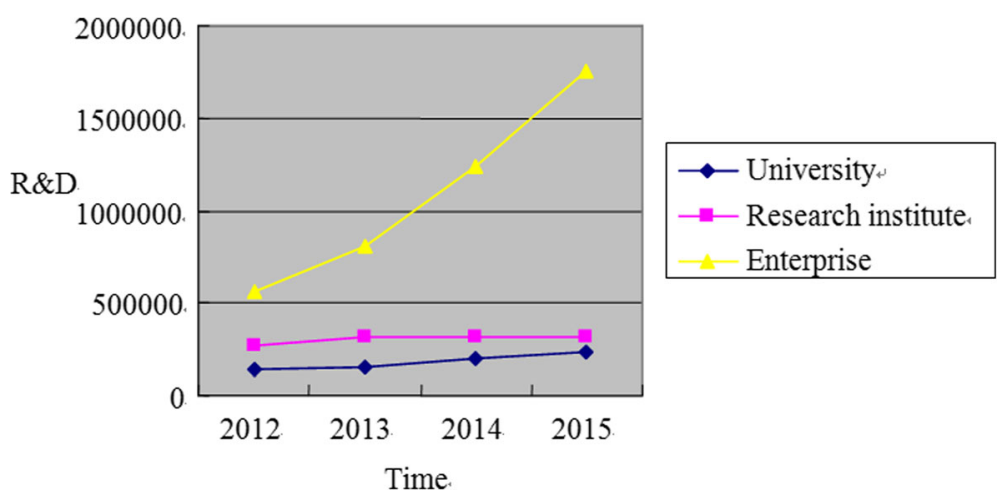

Fig. 10 R\&D block chain WEB from 2012 to 2015 
has received extensive attention from domestic and foreign scholars. By introducing a trust mechanism in P2P, we hope to solve the security problems it faces. This paper discusses the issues that need further study because of the latest research progress of the trust mechanism. However, because of the many similarities between P2P networks and human society, this causes trust mechanisms to be more complex. The research on trust mechanisms in P2P networks is also applicable to other computing environments, such as pervasive computing environments, ad hoc networks. With reference significance, the use of blockchain technology to develop a decentralized education system will help to break the monopoly of education rights that has been monopolized by schools or government agencies, so that education will become fully open, and an integrated education system will be formed with the participation of the whole people. In the future, the qualifications provided by education institutions such as schools and training institutions approved by government agencies to provide educational services, there will be more institutions and even individuals who assume the role of professional education service providers, and they will be open source, transparent, and based on the blockchain. Features such as non-derog ability can guarantee the authenticity and credibility of their educational process and results.

\section{Abbreviations \\ FP: File provider; GRA: Gray relational analysis; MLE: Maximum likelihood estimation}

\section{Acknowledgements}

This work was supported by the Fund Project of Hunan Social Sciences (grant No.15YBB031), the project of the humanities and social sciences fund of the Ministry of Education (grant No. 16YJAZH085), and the Fund Project of Hunan Social Sciences (grant No. 17YBA142).

\section{Funding}

The Fund Project of Hunan Social Sciences (grant No.15YBB031)

The project of the humanities and social sciences fund of the Ministry of Education (grant No. 16YJAZH085).

The Fund Project of Hunan Social Sciences (grant No. 17YBA142)

\section{Availability of data and materials}

The data generated and analyzed during this study are included in this published article, and its supplementary information isalso available from the corresponding author on reasonable request.

\section{Authors' contributions}

The manuscript was written through the contributions of all authors. All authors have given approval to the final version of the manuscript.

\section{Authors' information}

Qi Liu: Lecturer, Business School, Central South University. Her research interests include technological innovation, business administration. XiaoZou: Professor, Business School, Hunan University of Technology. Her research interests include business administration, logistics management.

\section{Competing interests}

The authors declare that they have no competing interests.

\section{Publisher's Note}

Springer Nature remains neutral with regard to jurisdictional claims in published maps and institutional affiliations.

Received: 21 November 2018 Accepted: 11 January 2019

Published online: 30 January 2019

References

1. Y. Guo, C. Liang, Blockchain application and outlook in the banking industry. Financ. Innov. 2(1), 24 (2016)

2. G. Li, S. Peng, C. Wang, et al., An energy-efficient data collection scheme using denoising autoencoder in wireless sensor networks. Tsinghua Sci. Technol. 24(1), 86-96 (2019)

3. M. Zheng, C. Liu, F. Liu, Average-consensus tracking of sensor network via distributed coordination control of heterogeneous multi-agent systems. IEEE Control Syst. Lett. 3(1), 132-137 (2019)

4. J. Sun, J. Yan, K.Z.K. Zhang, Blockchain-based sharing services: what blockchain technology can contribute to smart cities. Financ. Innov. 2(1), 26 (2016)

5. G. Yang, Y. Zhang, J. Yang, et al., Automated classification of brain images using wavelet-energy and biogeography-based optimization. Multimed. Tools Appl. 75(23), 15601-15617 (2016)

6. J. Yli-Huumo, D. Ko, S. Choi, et al., Where is current research on blockchain technology? - a systematic review. PLoS One 11(10), e0163477 (2016)

7. Schweizer A, Schlatt V, Urbach N, et al. Unchaining Social BusinessesBlockchain as the Basic Technology of a Crowdlending Platform. 2017

8. O.E. Williamson, The new institutional economics: taking stock, looking ahead. J. Econ. Lit. 38(3), 595-613 (2000)

9. V. Brătăşanu, Digital innovation the new paradigm for financial services industry. Theoret. Appl. Econ. 24, 83-94 (2017)

10. X. Luo, W. Zhong, X. Li, X. Guan, S. Albrecht, S. Reichert, J. Schmid, et al, Rigid graph-based three-dimension localization algorithm for wireless sensor networks. J. Syst. Eng. Electron. 29(5), 927-936 (2018)

11. R. Ribitzky, J.S. Clair, D.I. Houlding, et al., Pragmatic, interdisciplinary perspectives on blockchain and distributed ledger technology: paving the future for healthcare. Blockchain Healthc. Today (2018)

12. G. Yang, X. Deng, C. Liu, Facial expression recognition model based on deep spatiotemporal convolutional neural networks. J. Cent. South Univ. (Sci. Technol.) 47(7), 2311-2319 (2016)

13. H. Zhu, Z.Z. Zhou, Analysis and outlook of applications of blockchain technology to equity crowdfunding in China. Financ. Innov. 2(1), 29 (2016)

14. G. Yang, W. Tan, H. Jin, et al., Review wearable sensing system for gait recognition. Clust. Comput., 1-9 (2018)

15. T. Fujimoto, K. Shigeta, Y. Fukuyama, The research trends in game-based learning and open education. Educ. Technol. Res. 39(1), 15-23 (2016)

16. Z. Chen, Y. Li, Y. Wu, et al., The transition from traditional banking to mobile internet finance: an organizational innovation perspective-a comparative study of Citibank and ICBC. Financ. Innov. 3(1), 12 (2017)

17. P. Bauer, A. Hiba, B.Z. Daróczy, et al., Real flight demonstration of monocular image-based aircraft sense and avoid. ERCIM NEWS 110, $42-43$ (2017)

\section{Submit your manuscript to a SpringerOpen ${ }^{\odot}$ journal and benefit from:}

- Convenient online submission

- Rigorous peer review

- Open access: articles freely available online

High visibility within the field

- Retaining the copyright to your article

Submit your next manuscript at $>$ springeropen.com 